\title{
Menschenbilder in der (Medizin-)Ethik
}

\author{
Sigrid Graumann • Marianne Rabe
}

Online publiziert: 8. Februar 2011

(c) Springer-Verlag 2010

Viele ethische Kontroversen in der Medizin und im Gesundheitswesen beruhen implizit oder explizit auf vorgefassten, kulturell geprägten Bildern vom Menschen. Auch dem Handeln von Ärztinnen und Ärzten oder von Pflegenden liegen Menschenbilder zugrunde. Schon das Verständnis von Krankheit und Gesundheit ist durch anthropologische Präsuppositionen geprägt: Wird Krankheit beispielsweise als bloße Funktionsabweichung oder vielmehr als Wechselwirkung von organischen Störungen mit psychischen und sozialen Faktoren verstanden? Die medizinische Forschung konzentriert sich im Allgemeinen auf die Erforschung von kausalen Krankheitsursachen, hat also einen eher naturalistischen Blick auf Krankheit. Trifft dies auch für die Medizin im Ganzen zu? Dort gibt es als Gegenpole immerhin die Tradition der medizinischen Anthropologie (z. B. Viktor von Weizsäckers) und die Ganzheitlichkeitsbewegung, die auch in der Pflege ihren Niederschlag fand. Mit dem Verständnis von Medizin wandelt sich jeweils auch das Selbstverständnis der Gesundheitsberufe.

Die Verletzlichkeit und Endlichkeit des Menschen werden mittlerweile verstärkt thematisiert, bestimmen neue Schwerpunktsetzungen im Gesundheitswesen wie die Prävention oder die Palliativmedizin und erweitern damit das traditionelle Heilungsparadigma. Medizinethische Grundlagendebatten drehen sich oft um Grenzsituationen des Menschseins wie Leiden und Sterben, aber auch die unklaren und sich verändernden Grenzen zwischen Leben und Tod, und bringen damit anthropologische Bezugspunkte der Ethik in den Blick, die in letzter Zeit verstärkt Beachtung finden.

Die Zunahme anthropologischer Argumente und Denkfiguren in der Ethik und die kontroverse Diskussion über die Berechtigung derselben haben zur Themenstellung für die Jahrestagung 2009 in Berlin geführt. Sie wurde von Sigrid Graumann (heute Universität Oldenburg) und Marianne Rabe (Charité Gesundheitsakademie Berlin) als gemeinsames

\footnotetext{
S. Graumann $(\bowtie)$

Institut für Sozialwissenschaften, Carl von Ossietzky Universität Oldenburg,

Ammerländer Heerstr. 114-118,

26111 Oldenburg, Deutschland

E-Mail: sigrid.graumann@uni-oldenburg.de

M. Rabe

Charité - Gesundheitsakademie, Berlin, Deutschland
} 
Projekt der Charité und des Instituts „Mensch Ethik und Wissenschaften“ Berlin (IMEW) durchgeführt.

Ziel der Jahrestagung war es, das Verständnis der unterschiedlichen Menschenbilder, die die medizinethischen Debatten und Kontroversen prägen, und das ihnen jeweils zugrunde liegende Ethikverständnis zu klären und zu diskutieren. Das Themenheft spiegelt mit der Wiedergabe der Hauptvorträge nur einen Ausschnitt der lebendigen Diskussionen, die gerade auch in den Sektionen mit den Kurzvorträgen geführt wurden.

Mit dem Festvortrag des Historikers Valentin Groebner aus Luzern wurde die aktuelle Problematisierung der Kommerzialisierung bzw. Komodifizierung des menschlichen Körpers durch den Gewebe- und Organhandel aus historischer Sicht beleuchtet. Sein Beitrag macht deutlich, dass sich die tradierte Entgegensetzung einer vermeintlich guten christlichabendländischen Tradition, die die Komodifizierung des menschlichen Körpers verbietet, mit einem neuen, pervertierten Menschenbild der heutigen Zeit, das alle Grenzen überschreitet, nicht halten lässt. Groebner zeigt, dass der Handel mit menschlichen Körpern, Körperteilen und -substanzen im christlichen Mittelalter, obwohl tabuisiert, als Handel mit menschlichen Körpersubstanzen als Bestandteil von Arzneimitteln, mit Körperteilen als Reliquien für die Kirchen und Klöster sowie mit Leichen für die Anatomie sehr verbreitet war.

Theda Rehbock plädiert am Beispiel der Hirntodproblematik für eine explizite Reflexion der anthropologischen Bedingungen sowohl des medizinischen Handelns als auch der ethischen Begriffsbildung. Nur wenn eine auf biologische Aspekte verengte Sichtweise vermieden wird, indem durch Rekurs auf die menschliche Grundsituation alle relevanten Aspekte ins Auge gefasst würden, ließe sich die besondere Situation des Hirntods angemessen beschreiben. Nur im ,personalen Sinnhorizont der menschlichen Lebenspraxis“ ließen sich die klinische Hirntodfeststellung und relevante ethische Prinzipien wie Autonomie und Würde angemessen verstehen und diskutieren.

Die Bezugnahme auf ein Menschenbild, das die Funktion der Vermittlung zwischen deskriptiven und normativen Dimensionen des menschlichen Selbstverständnisses einnimmt, hält Marcus Düwell, wie er in seinem Beitrag ausführt, für höchst problematisch. Er argumentiert, dass die Formulierung eines umfassenden Verständnisses vom Menschen zwar sinnvoll sein kann, um ontologische Voraussetzungen moralischer Diskurse zu explizieren, die notwendige Begründung von Handlungsnormen aber nicht leisten kann.

Gesa Lindemann thematisiert in ihrem Beitrag, dass die heutige Vorstellung vom biologisch lebenden Menschen, der als „soziale Person“ in der Lage sei, verschiedene gesellschaftliche Ordnungen zu schaffen, historisch kontingent sei. Sie argumentiert, dass in anderen Zeiten auch zu Verstorbenen, Dämonen oder Tieren legitime soziale Beziehungen unterhalten werden konnten. Für die Konstitution sozialer Personen in der modernen Gesellschaft seien vier Grenzziehungen konstitutiv - am Anfang des Lebens, am Ende des Lebens, zwischen Mensch und Tier sowie zwischen Mensch und Maschine. Das aber würde für die Medizinethik bedeuten, dass die ethischen Fragen an den Grenzen des Lebens eine ganz grundsätzliche Bedeutung für die normative Ordnung moderner Gesellschaften hätten. Sie müssten daher in einem deutlich weiteren Horizont behandelt werden, als das heute oft der Fall sei.

Die in der Ethik diskutierten Fragen, welches Verständnis von Krankheit und Behinderung in Medizin und Medizinethik dominiert, wie die beiden Begriffe unterschieden werden sollten und welche anthropologischen Annahmen dabei explizit oder implizit eine Rolle spielen, spiegeln sich auch in der neuen Klassifikation von Behinderung der WHO, der sog. ICF (International Classification of Functioning, Disability and Health). Am Beispiel von Aufmerksamkeitsstörungen untersucht Marianne Hirschberg die Stärken und Schwächen 
dieser neuen Klassifikation, insbesondere was die Unterscheidung von Krankheit und Behinderung angeht.

Die Suche nach spirituellen Deutungen gehört ebenso zur menschlichen Grundsituation wie die Verwiesenheit auf Gemeinschaft und die grundsätzliche Verletzlichkeit des Menschen. Johannes Fischer zeigt am Beispiel eines Psalmtextes (Ps. 31), wie religiöse Wahrnehmung von Krankheit aussehen kann: Hier geht es nicht darum, Krankheit und Leiden mit Sinn zu versehen; indem sie vor Gott gebracht werden, werden sie in ein religiöses Bezugssystem gestellt, das dem Beter Distanz zur eigenen Angst und Verzweiflung ermöglicht. Übliche Sinndeutungen in Bezug auf Krankheit, wie Leiden als Strafe, als Probe und als Heilsweg, werden von Fischer kritisch diskutiert. Er folgert: „Wenn Religion etwas ermöglicht, dann vor allem dieses: mit Sinnlosigkeit leben zu können“.

Über den Workshop „Ethische Implikationen der Medizinischen Anthropologie Viktor von Weizsäckers“ berichten drei Beiträge von Mitgliedern der Viktor von Weizsäcker Gesellschaft, die den Workshop organisierten und durchführten. Rainer-M. E. Jacobi geht in seiner Einführung auf einige Grundbegriffe und Grundannahmen der Medizinischen Anthropologie von Weizsäckers ein.

Klaus P. G. Gahl skizziert die Umrisse der anthropologischen Medizin, die als ,Umgangslehre" die Beziehung zwischen hilfsbedürftigem Menschen und Helfer betont. Dabei werden einerseits die objektiven Daten der naturwissenschaftlichen Medizin nicht vernachlässigt, andererseits aber die verbreitete Engführung einer objektivistischen Betrachtung zurückgewiesen, die dem subjektiven Krankheitserleben keinen Raum gibt.

Hartwig Wiedebach schließlich formuliert den Entwurf einer ,pathischen Ethik“. Das Pathische ist ein Zentralbegriff in von Weizsäckers Medizinischer Anthropologie und beschreibt eine Haltung des Geschehenlassens gegenüber der Unverfügbarkeit des Lebens. Das ,pathische Urteil“ verhindert, dass solches Geschehenlassen nicht in ziellose Passivität abgleitet. Es bedient sich als Hilfsmittel der ,pathischen Kategorien“ und kann so zu einer hilfreichen Umgestaltung von Widerfahrnissen beitragen.

Die Tagungsleiterinnen freuen sich, dass dieses Themenheft einige wichtige Diskussionsbeiträge in der Debatte um Ethik und Anthropologie aufnimmt, und danken der Schriftleitung und Redaktion von Ethik in der Medizin sowie dem Springer-Verlag für die Möglichkeit dieser Publikation. 